MATHEMATICS OF COMPUTATION

Volume 66, Number 219, July 1997, Pages 1161-1168

S 0025-5718(97)00860-0

\title{
UNIVERSAL BINARY HERMITIAN FORMS
}

\author{
A. G. EARNEST AND AZAR KHOSRAVANI
}

\begin{abstract}
We will determine (up to equivalence) all of the integral positive definite Hermitian lattices in imaginary quadratic fields of class number 1 that represent all positive integers.
\end{abstract}

\section{INTRODUCTION}

The search for positive definite quaternary integral quadratic forms which represent all positive integers has a long and illustrious history, dating back to Lagrange's proof in 1770 that the form $x^{2}+y^{2}+z^{2}+w^{2}$ has this property. Such forms are referred to as universal in the contemporary literature. More generally, a positive integral quadratic form over a totally real number field is said to be universal if every totally positive integer of the field is represented by the form. While no universal positive binary quadratic forms exist, Maass [8] showed that the sum of three squares is universal over $\mathbf{Q}(\sqrt{5})$. In 1994, Chan, Kim and Raghavan [1] showed that among the real quadratic fields, only the fields $\mathbf{Q}(\sqrt{2}), \mathbf{Q}(\sqrt{3})$, and $\mathbf{Q}(\sqrt{5})$ admit universal ternary classic integral quadratic forms; all such forms are listed by the authors.

In this paper, we consider the analogous problem of finding universal positive definite Hermitian forms. It will be shown that over all imaginary quadratic fields, there exist only finitely many classes of universal binary positive definite Hermitian forms. All such forms will be determined for the imaginary quadratic fields of class number 1 ; i.e., the fields $\mathbf{Q}(\sqrt{m})$ where $m=-1,-2,-3,-7,-11,-19$, $-43,-67,-163$.

Computational methods were used to produce a list containing all potentially universal binary Hermitian forms, and all classes in their genera, over the nine imaginary quadratic fields of class number 1 . We now give a brief outline of the method used. First, an upper bound for the discriminant of a universal binary Hermitian form is determined for each of the fields. Next, inequalities are obtained via reduction theory for the coefficients of such forms. The potentially universal reduced forms are then listed and are screened for possible universality by determining whether the integers 1 through 5 are represented. This rough screening leaves thirteen candidates. Of these, six give rise via the trace mapping of Jacobson [4] to diagonal integral quaternary quadratic forms; their universality is established by appealing to known results for universality of such diagonal forms. For the remaining forms, all binary Hermitian forms of the given discriminant are computer-generated

Received by the editor May 15, 1996.

1991 Mathematics Subject Classification. Primary 11E39; Secondary 11E20, 11E41.

Research supported in part by a grant from the National Security Agency.

(C)1997 American Mathematical Society 
and separated into genera. The four candidates which are found in this way to have only one class in their genus are then known to be universal. The details and results of these computations are given in section 3 of this paper. The genera of the remaining three candidates contain two classes each, so establishing their universality requires further argument. Each of these genera is treated individually in the remaining sections of the paper. Ultimately, all the candidates that passed the initial screening are proven to be universal.

\section{Preliminaries}

Let $E$ be an imaginary quadratic field with nontrivial Q-involution ${ }^{-}$and ring of algebraic integers $\mathcal{O}$. A finitely generated $\mathcal{O}$-module $L$ is an Hermitian lattice over $\mathcal{O}$ if there is a nondegenerate Hermitian space $(V, H)$ over $E$ for which $E L=V$. All lattices considered here will be assumed to be integral and positive definite, in the sense that $H(x, y) \in \mathcal{O}$ for all $x, y \in L$ and $H(x)>0$ for all $x \neq 0$ (here and throughout the paper, $H(x, x)$ is abbreviated simply to $H(x))$. Note that it follows from these assumptions that $H(x) \in \mathbf{Z}$ for all $x \in L$. In this paper, the term Hermitian lattice will always refer to a positive definite integral Hermitian lattice over $\mathcal{O}$.

In the case that $\mathcal{O}$ is a principal ideal domain (i.e., $E$ is a field of class number 1 ), every Hermitian lattice is free and each choice of an $\mathcal{O}$-basis $\left\{v_{1}, \ldots, v_{n}\right\}$ for $L$ gives rise to a function $f: \mathcal{O}^{n} \rightarrow \mathbf{Z}$ defined by $f\left(x_{1}, \ldots, x_{n}\right)=H\left(\sum x_{i} v_{i}\right)=$ $\sum H\left(v_{i}, v_{j}\right) x_{i} \overline{x_{j}}$. Such a function will be referred to as an Hermitian form associated to $L$. In this way, isometry classes of Hermitian lattices on a fixed Hermitian space correspond to classes of Hermitian forms equivalent under invertible integral transformations.

Our primary interest here lies in the integers represented by Hermitian lattices. An integer $a$ is said to be represented by the Hermitian lattice $L$ if there exists $x \in L$ for which $H(x)=a$. In terms of an associated Hermitian form $f$, this is equivalent to the existence of $\alpha_{1}, \ldots, \alpha_{n} \in \mathcal{O}$ such that $f\left(\alpha_{1}, \ldots, \alpha_{n}\right)=a$. A major tool in our analysis of representation properties of Hermitian lattices will be a mapping which associates to a given Hermitian lattice $L$ a quadratic lattice $\tilde{L}$, having twice the rank of $L$, which represents the same integers as $L$. To each Hermitian space $(V, H)$, Jacobson [4] associated a symmetric bilinear space $\left(\tilde{V}, B_{H}\right)$ where $\tilde{V}$ denotes $V$ considered as a vector space over $\mathbf{Q}$ and

$$
B_{H}(x, y)=\frac{1}{2}[H(x, y)+H(y, x)]=\frac{1}{2} \operatorname{Tr}_{E / Q}(H(x, y)) .
$$

Analogously, associate to a Hermitian $\mathcal{O}$-lattice $L$ on $V$ the quadratic Z-lattice $\tilde{L}$ on $\tilde{V}$ obtained by viewing $L$ as a $\mathbf{Z}$-lattice on $\left(\tilde{V}, B_{H}\right)$. Then $L$ and $\tilde{L}$ represent the same integers, as $B_{H}(x, x)=\frac{1}{2} \operatorname{Tr}_{E / Q}(H(x, x))=H(x)$ for all $x \in L$. Now let $m$ be a squarefree negative integer such that $E=\mathbf{Q}(\sqrt{m})$, and write $\omega=\sqrt{m}$ if $m \equiv 2,3(\bmod 4)$ or $\omega=(1+\sqrt{m}) / 2$ if $m \equiv 1(\bmod 4)$. Then $\tilde{f}\left(x_{1}, y_{1}, \ldots, x_{n}, y_{n}\right)=$ $f\left(x_{1}+\omega y_{1}, \ldots, x_{n}+\omega y_{n}\right)$ is a quadratic form in $2 n$ variables corresponding to the lattice $\tilde{L}$.

\section{Generating possible universal binary Hermitian forms}

For the remainder of the paper, we consider only binary Hermitian forms and their corresponding quaternary quadratic forms. Let $f(x, y)=a x \bar{x}+b x \bar{y}+\bar{b} \bar{x} y+c y \bar{y}$, 
with $a, c \in \mathbf{N}, b=b_{1}+\omega b_{2}, b_{1}, b_{2} \in \mathbf{Z}$; the discriminant of $f$ is $D=a c-N_{E / Q}(b)$. Let $\tilde{D}$ be the determinant of the quaternary quadratic form $\tilde{f}$ associated to $f$ via the trace map as described in the preceding section. Then $D$ and $\tilde{D}$ are related by the equation $\tilde{D}=\left(D^{2} \operatorname{disc}(E / \mathbf{Q})^{2}\right) / 16$ (see [9]). By a result of Ross (see [12]), the determinant of a universal positive definite quaternary quadratic form does not exceed 861. Moreover, $f$ is a universal binary Hermitian form if and only if $\tilde{f}$ is a universal quaternary quadratic form. It follows that there are only finitely many classes of universal binary Hermitian forms over all imaginary quadratic fields, that the largest possible discriminant of a universal binary Hermitian form over $\mathbf{Q}(\sqrt{m})$ is $29,14,39,16,10,6,2,1$ for $m=-1,-2,-3,-7,-11,-19,-43,-47$, respectively, and that $\mathbf{Q}(\sqrt{-163})$ does not admit such a lattice.

From this point on, we will always assume that $E$ is one of the nine imaginary quadratic fields of class number 1 . Consequently, every class of positive definite binary Hermitian lattices over $E=\mathbf{Q}(\sqrt{m})$ contains a reduced form $f$ whose coefficients satisfy:

$$
a=\min f, \quad-\frac{1}{2} a \leq b_{1} \leq \frac{1}{2} a, \quad 0 \leq b_{2} \leq \frac{1}{2} a, \text { and } a \leq c
$$

(e.g., see [13]). The minimum of the corresponding quaternary quadratic form $\tilde{f}$ is no greater than $\sqrt{2} \tilde{D}^{1 / 4}$ (e.g., see [7, p. 294] Consequently, from the relationship between $D$ and $\tilde{D}$, we obtain the inequality

$$
a \leq \sqrt{2}(D(-\operatorname{disc}(E / \mathbf{Q}) / 4))^{1 / 2} .
$$

As $f$ is positive definite, $1 \leq a$, thus giving a finite range for $a$ for any given discriminant $D$. Thus, to set up a program to enumerate representatives from every class of binary Hermitian forms of discriminant $D$, it remains to find an upper bound for $c$ in terms of $D$ and $a$. Using the inequalities above, we get

$$
a c-\left(\frac{a^{2}}{4}(2+N(w))\right) \leq D \leq a c .
$$

Isolating $c$ in this inequality yields the desired upper bound

$$
c \leq \frac{D}{a}+\frac{a}{2}+N(w) \frac{a}{4} .
$$

In case the reduced binary form $f$ is universal, it must be that $a=1$. The inequalities above for the integers $b_{1}$ and $b_{2}$ then force $b_{1}=b_{2}=0$; that is, $f$ is a diagonal form. Consequently, the possible reduced universal binary Hermitian forms are of the form $x \bar{x}+D y \bar{y}$, where $D$ does not exceed the bound given above for the relevant field. For each of these forms the number of representations of the integers $n$ were determined for each of the integers 1 through 5. The computer program used for this purpose worked with the associated integral quaternary quadratic form $\tilde{f}$ and was based on the method of successively completing squares to diagonalize a multiple of this form over the ring of rational integers; it is then routine to find bounds on the four integer variables and count the number of integral vectors $x$ satisfying $\tilde{f}(x)=n$. If the number of representations of some integer in this range is zero, then $f$ cannot be universal. 
This screening method eliminates all but thirteen forms from the prior list. These forms are:

$$
\begin{array}{rll}
1)-5) & x \bar{x}+y \bar{y} \quad \text { in } \mathbf{Q}(\sqrt{m}) \quad \text { for } m=-1,-2,-3,-7,-11, \\
6)-11) & x \bar{x}+2 y \bar{y} \quad \text { in } \mathbf{Q}(\sqrt{m}) \quad \text { for } m=-1,-2,-3,-7,-11,-19, \\
12)-13) & x \bar{x}+3 y \bar{y} \quad \text { in } \mathbf{Q}(\sqrt{m}) \quad \text { for } \quad m=-1,-2 .
\end{array}
$$

The remainder of the paper is devoted to proving that the above forms are indeed universal.

The quaternary quadratic forms which correspond to the Hermitian forms 1), $2), 6), 7), 12$ ), and 13) are the diagonal forms:

$$
\begin{aligned}
\left.1^{\prime}\right) & x^{2}+y^{2}+z^{2}+w^{2}, \\
\left.2^{\prime}\right) & x^{2}+y^{2}+2 z^{2}+2 w^{2}, \\
\left.6^{\prime}\right) & x^{2}+y^{2}+2 z^{2}+2 w^{2}, \\
\left.7^{\prime}\right) & x^{2}+2 y^{2}+2 z^{2}+4 w^{2}, \\
\left.12^{\prime}\right) & x^{2}+y^{2}+3 z^{2}+3 w^{2}, \\
\left.13^{\prime}\right) & x^{2}+2 y^{2}+3 z^{2}+6 w^{2} .
\end{aligned}
$$

Each of these was shown to be universal by Ramanujan [10]; consequently, these six binary Hermitian forms are universal.

For the remaining seven candidates, it was first checked that the forms represent all positive integers locally with respect to all primes (here the notion of localization is that introduced by Shimura [11]; for a description of the local representation conditions the reader is referred to [3] and [4]). Then, utilizing the inequalities (1) and (2), all reduced forms with the same discriminant as each of the given forms were computer generated. These forms were then separated into equivalence classes, with the aid of the computations of representation numbers as described above. Finally, the inequivalent forms were separated into genera by checking their local invariants. In this way it was determined that the forms 3), 8), 4) and 10) each lie in a genus of a single class. As it is known that the forms in a genus collectively represent all integers represented by the genus, it follows that these four forms are also universal.

\section{The universality of The FORM $f=x \bar{x}+y \bar{y}$ IN $\mathbf{Q}(\sqrt{-11})$}

In $\mathbf{Q}(\sqrt{-11})$, the genus of $f=x \bar{x}+y \bar{y}$ consists of the class of $f$ and the class of $g=2 x \bar{x}+w \bar{x} y+\bar{w} x \bar{y}+2 y \bar{y}$. Using the trace map, we get the quaternary quadratic forms corresponding to $f$ and $g$, respectively:

$$
\begin{gathered}
\tilde{f}=x^{2}+3 y^{2}+z^{2}+3 w^{2}+x y+z w \\
\text { and } \quad \tilde{g}=2 x^{2}+6 y^{2}+2 z^{2}+6 w^{2}+2 x y+x z-5 x w+6 y z+3 y w+2 z w .
\end{gathered}
$$

The reduced form equivalent to $\tilde{g}$ is

$$
\tilde{g}_{R}=2 x^{2}+2 y^{2}+2 z^{2}+2 w^{2}+2 x y+x z+x w+y w+2 z w .
$$

Since equivalent forms represent the same integers, we will use $\tilde{g}_{R}$ in place of $\tilde{g}$. Straightforward computations show that $\tilde{f}(x+z, y+w, x-z, y-w)=2 \tilde{f}(x, y, z, w)$. So, in order to show that $\tilde{f}$ is universal, it suffices to show that $\tilde{f}$ represents all 
odd positive integers. So suppose $n$ is an odd positive integer represented by $\tilde{g}_{R}$. Completing squares yields the identity

$$
\begin{aligned}
8 \tilde{g}_{R}(x, y, z, w) & =(4 x+2 y+z+w)^{2}+(-y+2 z-2 w)^{2}+11(z+w)^{2}+11 y^{2} \\
& =r^{2}+s^{2}+11 t^{2}+11 u^{2}
\end{aligned}
$$

where $r$ and $t$ are of the same parity and $s$ and $u$ are of the same parity. We need to consider the following two cases:

I) $\quad r, t$ and $s, u$ have opposite parities,

II) $\quad r, t, s$, and $u$ are of the same parity.

We will show the latter is the only case that needs to be considered; first assume the former. Assume $r$ and $t$ are odd and $s$ and $u$ are even. Then $y$ must be even and $z+w$ must be odd, which implies that either $z$ or $w$ must be odd. Since $n$ is odd, and

$$
\begin{aligned}
n & \equiv x z+x w+y w \quad(\bmod 2) \\
& \equiv x(z+w)+y w \quad(\bmod 2),
\end{aligned}
$$

$x$ must also be odd. Suppose $z$ is odd, then consider the representation $(w, z, y, x)$ of $n$ by $\tilde{g}_{R}$ such that $r, s, t$, and $u$ are all odd. If $w$ is odd, then $(y, x,-z, z+w)$ yields a representation of $n$ by $\tilde{g}_{R}$ such that $r, s, t$, and $u$ are all odd.

Next assume $r$ and $t$ are even and $s$ and $u$ are odd. Then $y$ is odd and $z+w$ is even. Since $n$ is odd, $w$ must be odd, and so $z$ must be odd. If $x$ is odd, then consider the representation $(y, x,-z, z+w)$ of $n$ by $\tilde{g}_{R}$ such that $r, s, t$ and $u$ are all odd. If $x$ is even, then consider the representation $(w, z, y, x)$ of $n$ by $\tilde{g}_{R}$ such that $r, s, t$, and $u$ are all odd.

Thus we may assume that the representation of $n$ by $\tilde{g}_{R}$ leads to case II. First suppose $r, s, t$, and $u$ are all even. Then $y$ and $z+w$ must be even, which in turn implies that $n \equiv x(z+w)+y w(\bmod 2)$ is even. Now suppose $r, s, t$, and $u$ are all odd. There are two possibilities: $(r-t)+(s-u) \equiv 0$ or $2(\bmod 4)$. We observe that if $(r-t)+(s-u) \equiv 0(\bmod 4)$, then $(r-t)-(s-u) \equiv 0(\bmod 4)$ as well. Moreover, if $(r-t)+(s-u) \equiv 2(\bmod 4)$ then $(r-t) \pm(s+u) \equiv 0(\bmod 4)$.

Now assume that $n$ is represented by $\tilde{g}_{R}$. We will show that $n$ is represented by $\tilde{f}$. Consider the substitution

$$
x=\frac{r \mp s-t-u}{4}, y=\frac{t+u}{2}, z=\frac{r \pm s-t+u}{4} \text { and } w=\frac{t-u}{2},
$$

where the top signs are selected in the case $(r-t)+(s-u) \equiv 0(\bmod 4)$, and the bottom in the case $(r-t)+(s-u) \equiv 2(\bmod 4)$. Then $x, y, z$ and $w$ are integers such that $\tilde{f}(x, y, z, w)=n$.

\section{Universality OF THE FORM $x \bar{x}+2 y \bar{y}$ IN $\mathbf{Q}(\sqrt{-7})$}

Over $\mathbf{Q}(\sqrt{-7})$, the genus of $f=x \bar{x}+2 y \bar{y}$ consists of the class of $f$ and the class of $g=2 x \bar{x}-w \bar{x} y-\bar{w} x \bar{y}+2 y \bar{y}$. Using the trace map, we get the quaternary quadratic forms corresponding to $f$ and $g$, respectively:

$$
\begin{gathered}
\tilde{f}=x^{2}+2 y^{2}+2 z^{2}+4 w^{2}+x y+2 z w \\
\text { and } \quad \tilde{g}=2 x^{2}+4 y^{2}+2 z^{2}+4 w^{2}+2 x y-x z+3 x w-4 y z-2 y w+2 z w .
\end{gathered}
$$

The reduced form equivalent to $\tilde{g}$ is

$$
\tilde{g}_{R}=2 x^{2}+2 y^{2}+2 z^{2}+2 w^{2}+x y+x z+y w-z w .
$$


Since equivalent forms represent the same integers, we will use $\tilde{g}_{R}$ in place of $\tilde{g}$. Straightforward computations show that $\tilde{f}(2 y, x, 2 w, z)=2 n$. So in order to show $\tilde{f}$, and hence $f$, is universal, it suffices to show that $\tilde{f}$ represents all odd positive integers.

Suppose $n$ is an odd positive integer represented by $\tilde{g}_{R}$. Since $n$ is odd, and

$$
\begin{aligned}
n & \equiv x y+x z+y w-z w \quad(\bmod 2) \\
& \equiv x(y+z)+w(y-z) \quad(\bmod 2),
\end{aligned}
$$

$y$ and $z$ must have opposite parities, and $x$ and $w$ must have opposite parities. Without loss of generality, we may assume $z$ is odd, as $(x, z, y,-w)$ is another representation of $n$ by $\tilde{g}_{R}$. Now $n=f\left(x+w, y,-z, \frac{x+z-w}{2}\right)$, where $\left(x+w, y,-z, \frac{x+z-w}{2}\right)$ is an integral solution.

\section{The universality of The FOrm $f=x \bar{x}+2 y \bar{y}$ IN $\mathbf{Q}(\sqrt{-19})$}

Over $\mathbf{Q}(\sqrt{-19})$, the genus of the form $f=x \bar{x}+2 y \bar{y}$ consists of $f$ and the class of $g=3 x \bar{x}+(1+w) \bar{x} y+(1+\bar{w}) x \bar{y}+3 y \bar{y}$. Using the trace map, we get the quaternary quadratic forms corresponding to $f$ and $g$, respectively:

$$
\begin{gathered}
\tilde{f}=x^{2}+5 y^{2}+2 z^{2}+10 w^{2}+x y+2 z w \\
\tilde{g}=3 x^{2}+15 y^{2}+3 z^{2}+15 w^{2}+3 x y+3 x z-8 x y+11 y z+15 y w+3 z w .
\end{gathered}
$$

The reduced forms equivalent to $\tilde{f}$ and $\tilde{g}$ are

$$
\tilde{f}_{R}=x^{2}+2 y^{2}+5 z^{2}+10 w^{2}+x z+2 y w
$$

and $\quad \tilde{g}_{R}=3 x^{2}+3 y^{2}+5 z^{2}+5 w^{2}+3 x y+2 x z+2 x w-y z+3 y w+4 z w$,

respectively. Since equivalent forms represent the same integers, we will freely use either of the pairs of forms where convenient.

To show that $\tilde{f}$, and hence $f$, is universal, we first make several reductions. Straightforward computations show that the identities

$$
\begin{gathered}
\tilde{f}(2 z, 2 w, x, y)=2 \tilde{f}(x, y, z, w) \quad \text { and } \\
\tilde{f}_{R}(x+2 z+2 w, x-z-w, y-2 w, y+w)=3 \tilde{f}_{R}(x, y, z, w)
\end{gathered}
$$

hold. So, in order to show that $\tilde{f}$ is universal, it suffices to show that $\tilde{f}$ represents all odd positive integers not divisible by 3 . So in the remainder of the argument we assume that $n$ is such an integer.

Completing squares yields the identities

$$
\begin{aligned}
12 \tilde{g}_{R}(x, y, z, w) & =(6 x+3 y+2 z+2 w)^{2}+2(2 y-3 z+3 w)^{2}+19 y^{2}+38(z+w)^{2} \\
& =r_{1}^{2}+2 s_{1}^{2}+19 t_{1}^{2}+38 u_{1}^{2}
\end{aligned}
$$

and

$$
\begin{aligned}
12 \tilde{g}(x, y, z, w) & =(6 x+3 y+3 z-8 w)^{2}+2(2 z+w)^{2}+19(3 y+z+2 w)^{2}+38 w^{2} \\
& =r_{2}^{2}+2 s_{2}^{2}+19 t_{2}^{2}+38 u_{2}^{2}
\end{aligned}
$$


For $i=1,2$, equations (1) and (2) imply $2 \mid\left(r_{i}^{2}+19 t_{i}^{2}\right)$; so $r_{i}$ and $t_{i}$ are of the same parity. Moreover, $r_{i}^{2}+2 s_{i}^{2}+3 t_{i}^{2}+2 u_{i}^{2} \equiv 0(\bmod 4)$. Since $r_{i}^{2}+3 t_{i}^{2} \equiv 0(\bmod 4)$ whenever $r_{i}$ and $t_{i}$ are of the same parity, it follows that $2 s_{i}^{2}+2 u_{i}^{2} \equiv 0(\bmod 4)$; hence, $s_{i}$ and $u_{i}$ are of the same parity. Finally, $r_{i}^{2}+2 s_{i}^{2}+t_{i}^{2}+2 u_{i}^{2} \equiv 0(\bmod 3)$. This relation is satisfied only in the following cases:

$$
\begin{aligned}
& \text { I) } r_{i} \equiv s_{i}(\bmod 3) \text { and } t_{i} \equiv u_{i}(\bmod 3) \\
& \text { II) } r_{i} \equiv s_{i}(\bmod 3) \text { and } t_{i} \equiv 2 u_{i}(\bmod 3) \\
& \text { III) } \quad r_{i} \equiv 2 s_{i}(\bmod 3) \text { and } t_{i} \equiv u_{i}(\bmod 3) \\
& \text { IV) } \quad r_{i} \equiv 2 s_{i}(\bmod 3) \text { and } t_{i} \equiv 2 u_{i}(\bmod 3) \\
& \text { V) } \quad r_{i} \equiv u_{i} \equiv 0(\bmod 3), s_{i} \not \equiv 0(\bmod 3) \text { or } \\
&
\end{aligned}
$$

Now assume that $n$ is represented by $\tilde{g}$ (equivalently, by $\tilde{g}_{R}$ ). We next show that if this representation leads to any of the cases I) - IV) above for the representation of $12 n$, then $n$ is represented by $\tilde{f}$. In cases I) and II), consider the substitution

$$
x=\frac{r_{i}+2 s_{i}-t_{i} \mp 2 u_{i}}{6}, y=\frac{t_{i} \pm 2 u_{i}}{3}, z=\frac{r_{i}-s_{i}-u_{i} \pm t_{i}}{6}, \text { and } w=\frac{u_{i} \mp t_{i}}{3}
$$

where the top signs are selected in case I), the bottom in case II). In cases III) and IV), consider the substitution

$$
x=\frac{2 s_{i}-r_{i}-t_{i} \mp 2 u}{6}, y=\frac{t_{i} \pm 2 u_{i}}{3}, z=\frac{r_{i}+s_{i}-u_{i} \pm t_{i}}{6}, \quad \text { and } w=\frac{u_{i} \mp t_{i}}{3}
$$

where the top signs are selected in case III), the bottom in case IV). Then $x, y, z$ and $w$ are integers such that $\tilde{f}(x, y, z, w)=n$.

Finally we assume that the known representation of $n$ by $\tilde{g}_{R}$ leads to case $\left.\mathrm{V}\right)$. Suppose first that the representation $(x, y, z, w)$ in equation (1) leads to $r_{1} \equiv u_{1} \equiv$ $0(\bmod 3), s_{1} \not \equiv 0(\bmod 3)$ and $t_{1} \not \equiv 0(\bmod 3)$. Then we have $z+w \equiv 0(\bmod 3)$ and $y \not \equiv 0(\bmod 3)$. Without loss of generality we may assume that $z \not \equiv 0(\bmod 3)$, for otherwise $u_{1} \equiv 0(\bmod 3)$ will imply $w \equiv 0(\bmod 3)$, and thus $n \equiv 0(\bmod 3)$, contrary to our restriction on $n$. We may also assume that $x \not \equiv 0(\bmod 3)$, for otherwise we may consider $(x+y,-y, w, z)$, for which $\tilde{g}_{R}(x+y,-y, w, z)=n$ and $x+y \not \equiv 0(\bmod 3)$. Now $\tilde{g}(-x-y+2 z, w, x-z-2 w, z)=n$, which leads via equation (2) to $r_{2}=-3 x-6 y+z-3 w, s_{2}=2 x-z-4 w, t_{2}=x+z+w$ and $u_{2}=z$. Since $x \not \equiv 0(\bmod 3)$, it follows that $s_{2} \not \equiv 0(\bmod 3)$ and $t_{2} \not \equiv 0(\bmod 3)$. Furthermore, $z \not \equiv 0(\bmod 3)$ will imply that $r_{2} \not \equiv 0(\bmod 3)$ and $u_{2} \not \equiv 0(\bmod 3)$. As case $\left.\mathrm{V}\right)$ is not satisfied for this representation of $n$ by $\tilde{g}, n \rightarrow \tilde{f}$ follows from one of the cases I)-IV). Now suppose that the representation $(x, y, z, w)$ of $n$ by $\tilde{g}_{R}$ leads in equation (1) to $s_{1} \equiv t_{1} \equiv 0(\bmod 3), r_{1} \not \equiv 0(\bmod 3)$ and $u_{1} \not \equiv 0(\bmod 3)$. Then $y \equiv 0(\bmod 3)$ and $z+w \not \equiv 0(\bmod 3)$. Without loss of generality we may assume $z \not \equiv 0(\bmod 3)$, for otherwise we may consider the representation $(x+y,-y, w, z)$ of $n$ by $\tilde{g}_{R}$ with $w \not \equiv 0(\bmod 3)$. Again consider the representation $\tilde{g}(-x-y+2 z, w, x-z-2 w, z)=n$ with the corresponding $r_{2}, s_{2}, t_{2}, u_{2}$ as above. Again $z \not \equiv 0(\bmod 3)$ will imply 
$r_{2} \not \equiv 0(\bmod 3)$. We may further assume that $x+y+z \not \equiv 0(\bmod 3)$ for otherwise

$$
\begin{aligned}
n & \equiv 5 z^{2}+5 w^{2}+2 x z+2 x w+4 z w \quad(\bmod 3) \\
& \equiv 3\left(z^{2}+w^{2}\right)+2(z+w)^{2}+2 x(z+w) \quad(\bmod 3) \\
& \equiv 2(z+w)(z+w+x) \quad(\bmod 3) \\
& \equiv 0(\bmod 3),
\end{aligned}
$$

contrary to our restriction on $n$. Finally, $x+z+w \not \equiv 0(\bmod 3)$ will imply $t_{1} \not \equiv$ $0(\bmod 3)$ and $s_{1} \not \equiv 0(\bmod 3)$. As before $n \rightarrow \tilde{f}$ follows from one of cases $\left.\left.\mathrm{I}\right)-\mathrm{IV}\right)$. This completes the proof.

As a final note, we observe that the ternary section of the form $\tilde{f}$ obtained by setting $w=0$ is a ternary quadratic form which Kaplansky [6] has recently conjectured to represent all positive odd integers.

\section{REFERENCES}

[1] W. Chan, M. H. Kim and S. Raghavan, Ternary universal integral quadratic forms over real quadratic fields, preprint.

[2] L. E. Dickson, Quaternary quadratic forms representing all integers, Amer. J. Math. 49 (1947), 39-56.

[3] L. J. Gerstein, Classes of definite Hermitian forms, Amer. J. Math. 100 (1978), pp. 81-97. MR 57:5946

[4] N. Jacobson, A note on hermitian forms, Bull. Amer. Math. Soc. 46 (1940), pp. 264-268. MR 1:325d

[5] A. A. Johnson, Integral representations of hermitian forms over local fields, J. Reine Angew. Math. 229 (1968), pp. 57-80. MR 37:1348

[6] I. Kaplansky, Ternary positive quadratic forms that represent all odd positive integers, Acta Arith. 70 (1995), pp. 209-214. MR 96b:11052

[7] C. G. Lekkerkerker, Geometry of Numbers, North-Holland, Amsterdam-London, 1969. MR 42:5915

[8] H. Maass, Über die Darstellung total positiver Zählen des Korpers $R(\sqrt{5})$ als Summe von drei Quadraten, Abh. Math. Sem. Hamburg, 14, (1941), pp. 185-91. MR 3:163a

[9] J. P. Prieto-Cox, Representation of positive definite Hermitian forms, Ph.D. Dissertation, Ohio State University (1990).

[10] S. Ramanujan, On the expression of a number in the form $a x^{2}+b y^{2}+c z^{2}+d u^{2}$, Proc. Cambridge Phil. Soc. 19 (1917), 11-21.

[11] G. Shimura, Arithmetic of unitary groups, Ann. of Math. 79 (1964), pp. 369-409. MR 28:2104

[12] M. F. Willerding, Determination of all classes of positive quaternary quadratic forms which represent all (positive) integers, Bull. Amer. Math. Soc. 54 (1948), pp. 334-337. MR 9:571e

[13] F. Z. Zhu, On the classification of positive definite unimodular hermitian forms, Chinese Sci. Bull. 36 (1991), 1506-1511. MR 93a:11027

Department of Mathematics, Southern Illinois University, Carbondale, Illinois $62901-4408$

Department of Mathematics, Southern Illinois University, Carbondale, Illinois 62901-4408

Current address: Department of Mathematics, University of Wisconsin, Oshkosh, Oshkosh, Wisconsin 54901-8631 